\title{
P-0764 Diabetic ketoacidosis - characteristics and precipitating factors in type 2 diabetic patients admitted in ICU
}

G. Ditu $^{1}$, M. Bodnarescu 1 , A. Pantea Stoian², C. Serafinceanu ${ }^{1,2}$, V. Elian ${ }^{1}$

${ }_{1}$ INDNBM N. Paulescu, Bucharest, Romania.

2UMF Carol Davila, Bucharest, Romania.

\section{Background}

Worldwide annu-al incidence of ketoacidosis (DKA) ranges from 4.6 to 8 cases per 1,000 pa-tients with diabetes, with a mortality rate of up to $10 \%$. Most cases of DKA are triggered by infection followed by cessation of insulin treatment. Affecting mainly Type 1 diabetes patients, the precipitating factors for DKA and its clinical pathophysiology in Type 2 diabetes (T2D) are less clear. Little is known about DKA in T2D in Romania.

Aim

Our study aims to assess the prevalence, precipitating factors and clinical characteristics of T2D patients with DKA who were admitted to the intensive care unit of the National Institute of Diabetes in Romania.

\section{Methods}

This was a retrospective study that included adult T2D patients admitted for DKA between January 2013 and October 2016. Patients diagnosed with hyperosmolar hyperglicemic state (HHS) were not included. The initial laboratory evaluation included measurement of blood glucose, serum creatinine, ketone bodies, and electrolytes (with the anion gap), osmolality, urinalysis, as well as initial arterial blood gases and blood count.

\section{Results}

We analized 106 patients (58.49\% women) with T2D, mean age $64,6 \pm 12$ years. At admission, $39.6 \%$ patients were treated with an insulin regimen, while $60.4 \%$ used a non-insulin treatment before admission or had no treatment.

Infection was the main precipitating factor of DKA (40.6\%) followed by new onset of T2D $(24.5 \%)$, antidiabetic treatment cessation (21.7\%) and other causes (22.65\%) including cardiovascular events, major surgery, pancreatitis, and trauma. Among patients who have stopped their medication, $18.8 \%$ were treated with insulin. Kussmaul breathing was present in $56.6 \%$ of the patients. Initial mean blood glucose was $615 \pm 270$ $\mathrm{mg} / \mathrm{dL}$, mean osmolality was $306,3 \pm 23,7 \mathrm{mOsm} / \mathrm{L}$, mean $\mathrm{pH}$ was $7,164 \pm 0,14$ and mean $\mathrm{HCO}^{3}-$ was $10 \pm 4,3 \mathrm{mmol} / \mathrm{L}$. Ketonuria was present in $70.1 \%$ of the patients. Potassium had a mean value $4,5 \pm 0,9 \mathrm{mmol} / \mathrm{L}, 13.59 \%$ patients had hypokalemia and $26.21 \%$ had hyperkalemia at hospital presentation. Overall, majority of cases $(54.72 \%)$ were with moderate DKA based on international criteria. Severe DKA was identified in $15.09 \%$.

\begin{tabular}{ll}
\hline Factor & Type 2 diabetes \\
\hline $\mathrm{N}$ & 106 \\
$\mathrm{Age}$ & $64,6 \pm 12 \mathrm{yr}$ \\
New onset/ Previous diabetes & $26 / 106(24,5 \%)$ \\
\hline Self cessation of therapy & $23 / 106$ from which 20 cases \\
& treated with IT \\
Infection & $41 / 106$ \\
Kussmaul breathing & $60(56,6 \%)$ \\
Mean serum glucose & $615 \pm 270 \mathrm{mg} / \mathrm{dl}$ \\
Ketonuria & $73(70.1 \%)$ \\
Mean pH & $7,164 \pm 0,14$ \\
Mean pCo2 & $24,7 \pm 7,2$ \\
Mean HCO3- & $10 \pm 4,3 \mathrm{mmol} / \mathrm{I}$ \\
Mean Sodium & $132,5 \pm 9,2$ \\
Mean Potassium & $4,5 \pm 0,9$ \\
Serum osmolarity & $306,3 \pm 23,7$ \\
\hline
\end{tabular}

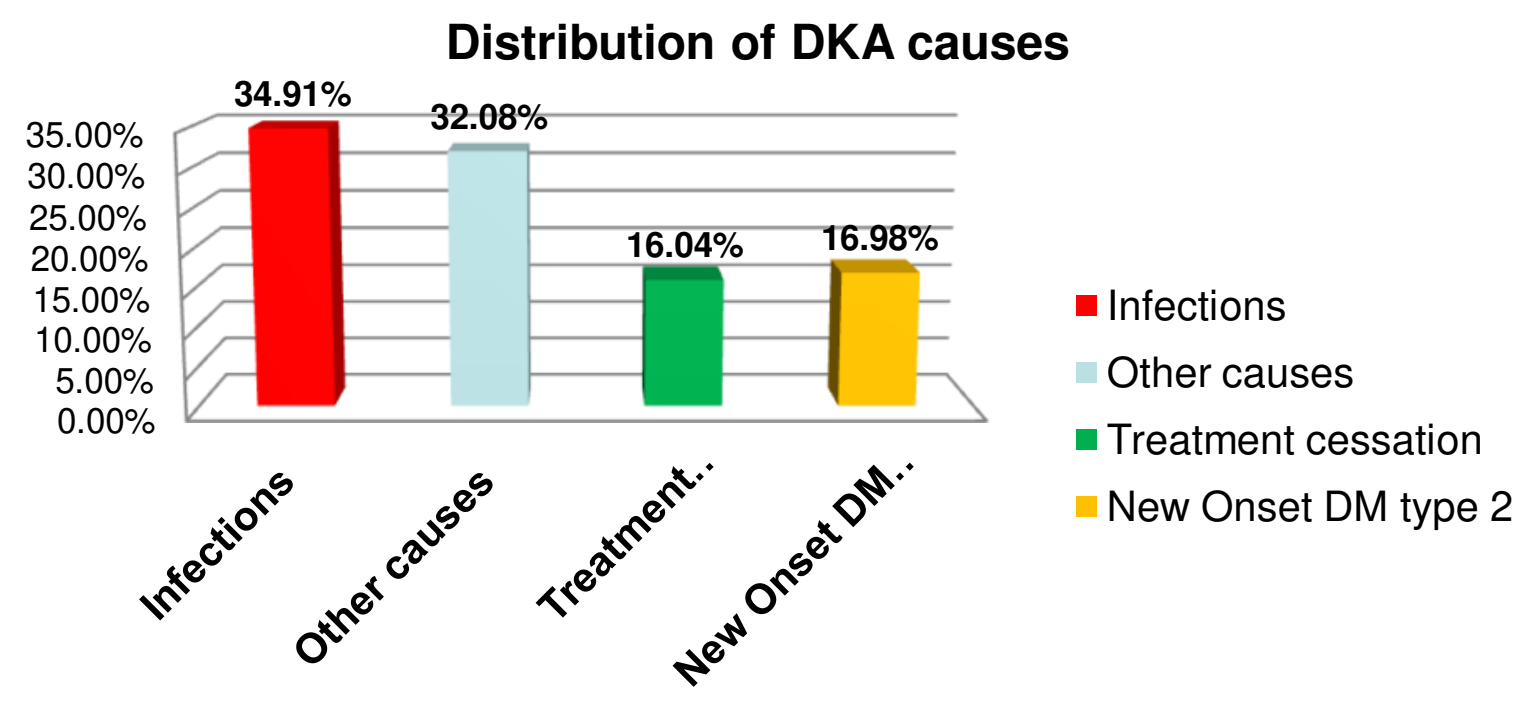

Patient distribution by the presence of Kussmaul breathing

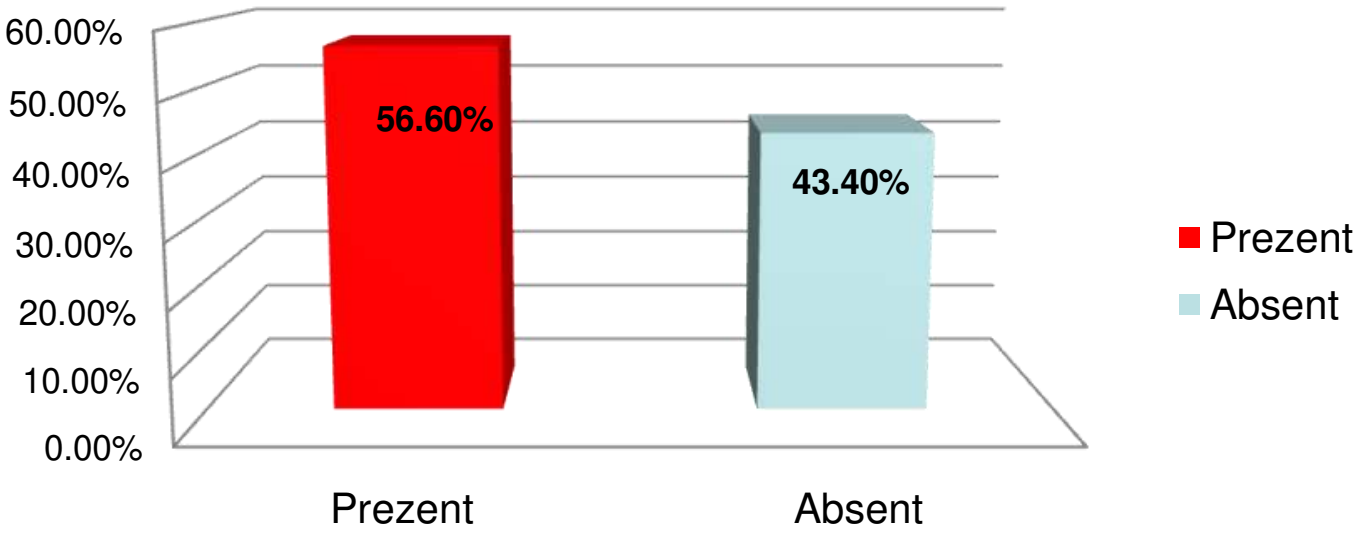

Patient Distribution by potassium value

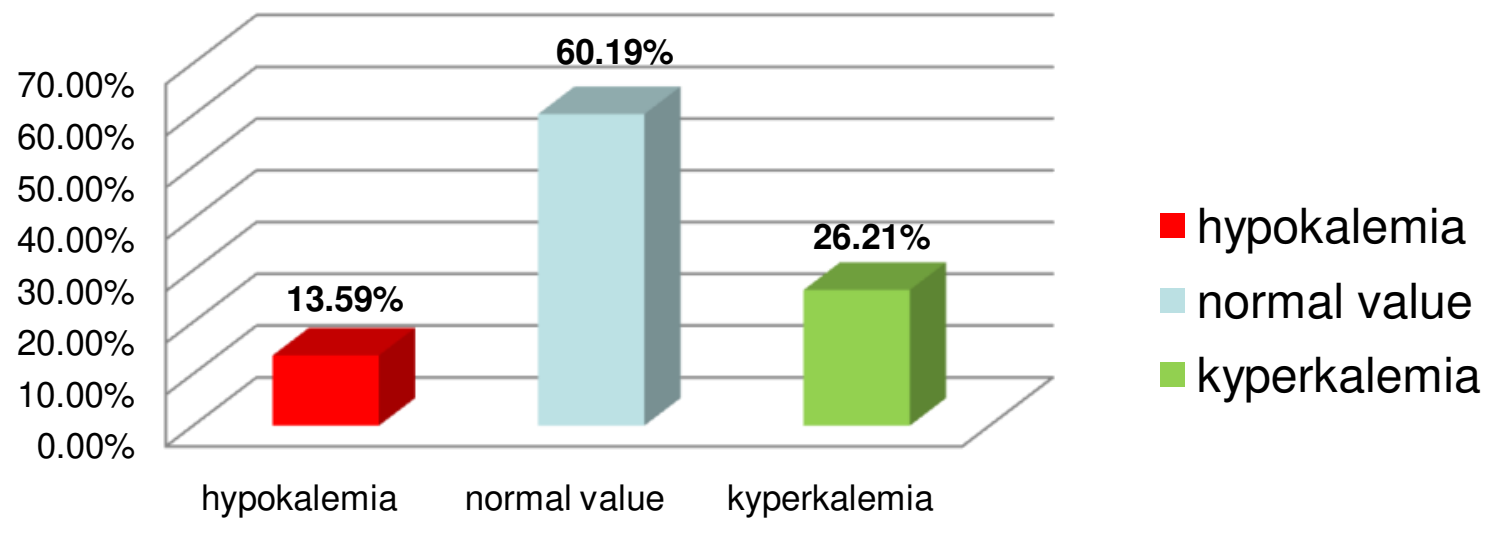

Potassium value

Patient distribution by DKA severity

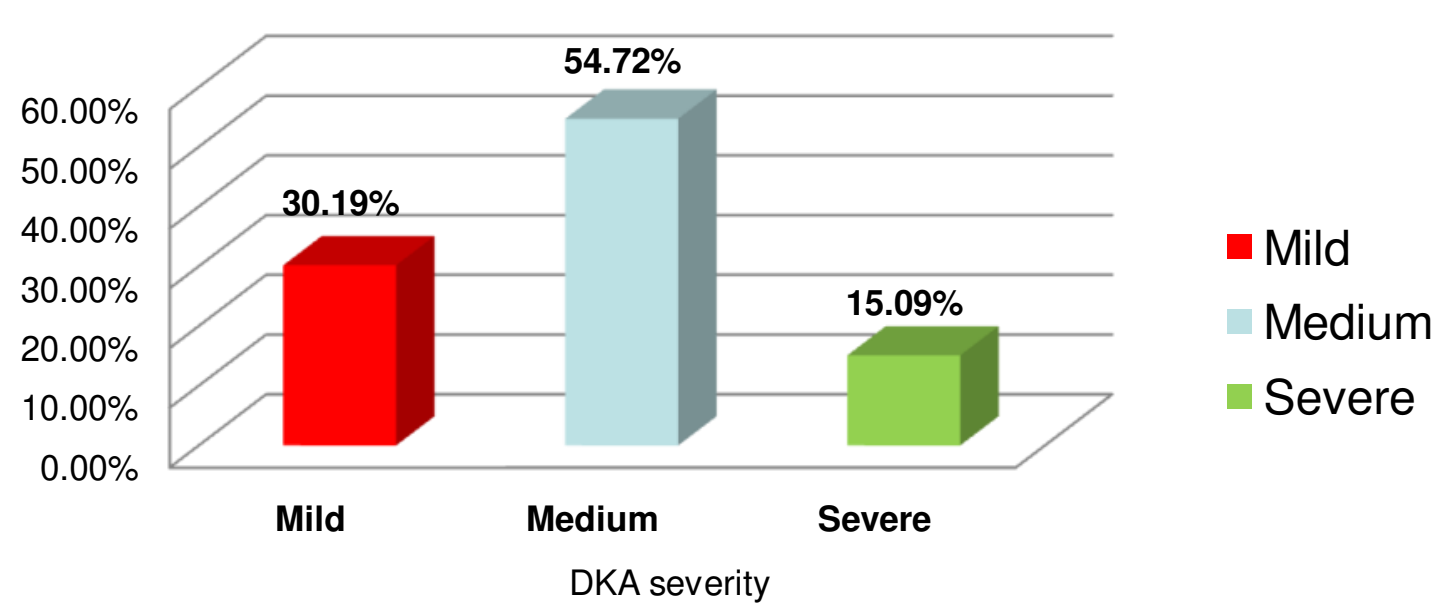

\section{Discussion}

In clinical practice, the patients at risk might benefit from earlier introduction and intensification of insulin therapy to avoid DKA and other complications of T2D. The high percentage of severe DKA at presentation observed in our analysis is associated mainly with infectious complications of T2D patients. Although we collected limited information regarding diabetes medication, our results show that treatment adherence, especially at insulin regimens, need particular consideration in diabetic patients. It is important to know the particularities of these patients and the most common causes of hospital admission for DKA in T2D patients for a rapid and successful clinical management. 